Ref.TH.3196-CERN

\title{
GAUGE INVARIANT VARIATIONAL STUDY OF THE HAMILTONIAN
}

U(1) AND $Z_{N}$ MODEL AND CRITICAL SPACE-TIME DIMENSIONALITY

C. Omero ${ }^{*}$

CERN -- Geneva

\section{ABSTRACT}

Using a gauge invariant variational formalism we find in $D=4$ dimensions a first order phase transition for $Z_{2,3,4}$ and two second ones for $Z_{N}$ with $N \geq 5$. At $D=3.2$ the transitions of $Z_{2}$ and $Z_{4}$ become of second order, whereas that of $Z_{3}$ for $D \geq 3$ remains of first order. For $D>4$ for all $N$ the transition in $Z_{N}$ is first order. In the $U(1)$ case for $3<D<4$ there is a second order transition so that the passage to the continuum limit is allowed, whereas for $D>4$ there is only a first order transition wi thout any long range correlation length; $\mathrm{D}=4$ appears therefore as the critical space-time dimensionality under which the theory exists in the continuum in agreement with the usual criterion of renormalizability. For $D<3$ the $U(1)$ model is always in the confining phase.

\footnotetext{
*) Permanent address: Istituto di Fisica Teorica and INFN, Trieste, Italy.
} 
It has recently been confirmed that Lagrangian mean field $\left.{ }^{1}, 2\right)$ and Hamiltonian variational techniques ${ }^{3), 4)}$ can be successfully employed to deal with gauge theories giving results in agreement with theoretical and numerical knowledge. The advantage of using a variational Hamiltonian approach is twofold: firstly there is a correspondence between the simplest variational ansatz and usual Lagrangian mean field approach, but also more elaborate variational states based on physical intuition are at our disposal as has been shown in Refs. 5) and 6). Secondly, but of much more relevance, gauge invariance can be implemented directly and in a very straightforward way, and this turns out to be essential in giving all the physical features, as has been seen for the gauge Higgs Ising system ${ }^{7}$.

In this letter we make use of the Hamiltonian technique in order to examine the $\mathrm{Z}_{\mathrm{N}}$ and the $\mathrm{U}(\mathrm{I})$ models in any Euclidean dimension $\mathrm{D} \gtrsim 2$.

Let us therefore consider the Hamiltonian for the $\mathrm{Z}_{\mathrm{N}}$ model:

$$
H_{Z_{N}}=-\sum_{\text {Rimkes }}\left(P_{e}+P_{e}^{+}\right) / 2-\lambda_{N} \sum_{\text {plag. } .5}\left(\left(Q Q Q^{+} Q^{+}\right)_{p}+\text { h.c. }\right) / 2
$$

where $\lambda_{\mathrm{N}}$ is the coupling constant having an inverse temperature character and $P$ and $Q$ are operators defined on the links of the system satisfying the $Z_{N}$

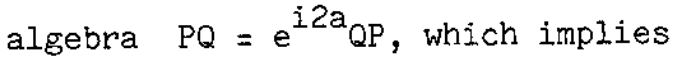

$$
\begin{array}{ll}
Q|q\rangle_{Q}=e^{i q 2 \pi / N}|q\rangle_{Q}, & P|q\rangle_{Q}=|q-1\rangle_{Q} \\
P|r\rangle_{P}=e^{i r 2 \pi / N}|r\rangle_{P}, & Q|r\rangle_{P}=|r+1\rangle_{P} .
\end{array}
$$

The Hamiltonian is invariant with respect to the local gauge transformation

$$
\left(G_{x}\right)^{\mu} H\left(G_{x}^{+}\right)^{\mu}=H \quad, G_{x}=\prod_{e \in X} P_{e}^{\eta_{e}}, \mu=0,1 \ldots N-1
$$

where the operator $G_{v}$ is the product of all the $P_{\ell}$ operators defined on the links $\&$ with orientation $\eta_{l}= \pm \pm$ belonging to the site $x$. If we take the limit $N \rightarrow \infty$ and identify $P=e^{i(2 \pi / N) L}$, and $Q=e^{i \theta}=U$ we have that

$$
\frac{N^{2}}{2 \pi^{2}} \cdot H_{Z_{N}} \rightarrow H_{U(1)}=\sum_{\text {eimks }} L_{e}^{2}-\lambda_{U(1)} \sum_{\text {plaq.s }}\left(\left(U U U^{\prime}\right)_{p}+h \cdot c .\right) / 2
$$

where $\lambda_{U(1)}=\lim \left(N^{2} / 2 \pi^{2}\right) \lambda_{Z_{N}}$ is the coupling constant of $U(1)$ and $\theta$ and $\mathrm{L}$ are the usual phase and angular momentum operators. 
In this work, in order to avoid numerical problems in angular integrations, the $U(1)$ theory will be recovered as a limit of $Z_{N}$. In order to build a trial ground state for the Hamiltonian we consider a state interpolating between the low and high $\lambda$ situations. This state, if we disregard only in the first instance the problem of gauge invariance, is conveniently expressed in terms of the eigenstates (2) of $P$ :

$$
|0\rangle=\prod_{\text {einies }}\left[\sum_{n=0}^{N-1} c_{n}^{1 / 2}(\beta)|n\rangle_{p}\right]_{e}=\prod_{l}|\beta\rangle_{e}
$$

It depends on the variational parameter $\beta=\beta(\lambda)$ and $c_{n}(\beta)$ are such that at high temperature (low $\beta$ ). $C_{n}(\beta(0)) \sim \delta_{\text {no }}$, and therefore in this limit the ansatz coincides with the eigenstate of $P$ with a higher real part, which minimizes the Hamiltonian and corresponds to a disordered state since $P^{\langle 0|Q| O\rangle_{P}}=0$. At low temperature (high $\lambda$ ) one chooses $c_{n}(\beta(\infty))=1$, so that the state corresponds to the zero eigenvalue of $Q|O\rangle_{Q}$, which is just one of the $N$ degenerate possible ordered states of the system.

A gauge invariant trial state $|\phi\rangle$ is obtained by just symmetrizing |0> with respect to all possible gauge transformations:

$$
|\phi\rangle=P_{G . I .}|0\rangle=\prod_{x}\left(\sum_{M_{x}=0}^{N-1} G_{x}^{\mu_{x}}\right) / N|0\rangle, \quad P_{G . I .}^{2}=P_{G . I}
$$

The main task now is to compute and minimize the variational energy

$$
E_{\phi}=\frac{1}{N_{\text {eimes }}}\langle\phi|H| \phi\rangle /\langle\phi \mid \phi\rangle
$$

For this purpose we first evaluate the norm $\langle\phi / \phi\rangle$ which, by straightforward calculation, turns out to be

$$
\begin{aligned}
\langle\phi \mid \phi\rangle & =\langle 0 \mid \phi\rangle=\sum_{\left\{M^{\prime}\right\}} \prod_{\left\{x x^{\prime}\right\}}\left[\sum_{m=0}^{N-1} c_{n}(\beta) \cos ((\mu-\mu) 2 \pi n / N)\right] \\
& =\sum_{\{M \times\}} \prod_{\left\{x^{\prime}\right\}} W\left(\cos \left(\tilde{\mu}-\tilde{\mu}^{\prime}\right), \beta\right)
\end{aligned}
$$

which is the sum over all possible local gauge transformations $\left\{\mu_{x}\right\}$ weighted with a Boltzmann factor corresponding to an effective generalized $\mathrm{Z}_{\mathrm{N}}$ model; $\left\{x^{\prime}\right\}$ are neighbouring sites and $\tilde{\mu}$ is an abbreviated version of $\mu 2 \pi / N$. If we choose 


$$
c_{n}(\beta)=\sum_{k=-\infty}^{+\infty} e^{-(n+k N)^{2} / 4 \beta}
$$

we recover the usual Villain model having as inverse temperature the variational parameter B. Choosing

$$
\cos (\beta)=\frac{1}{N} \sum_{\mu=0}^{N-1} \cos n \tilde{\mu} e^{\beta \cos \tilde{\mu}}
$$

we get the Wilson-Boltzmann factor

$$
W\left(\cos \left(\tilde{\mu}-\tilde{\mu}^{\prime}\right)_{l} \beta\right)=e^{\beta \cos \left(\tilde{\mu}-\tilde{\mu}^{\prime}\right)}
$$

In addition:

$$
\begin{aligned}
& \left\langle\phi\left|P_{\ell_{12}}\right| \phi\right\rangle=\sum_{\left\{\mu_{x}\right\}} \frac{W\left(\cos \left(\tilde{\mu}_{1}+\tilde{\mu}-\tilde{\mu}_{2}\right), \beta\right)}{W\left(\cos \left(\tilde{\mu}_{1}-\tilde{\mu}_{2}\right), \beta\right)} \prod_{\left\{x x^{\prime}\right\}} W\left(\cos \left(\tilde{\mu}-\tilde{\mu}^{\prime}\right)_{1} \beta\right) \\
& \left\langle\phi\left|Q_{\ell_{12}} Q_{e_{23}} Q_{\ell_{34}} Q_{e_{41}}\right| \phi\right\rangle=\sum_{\left\{\mu_{x}\right\}}\left\{\prod_{k=1,4} e^{i \frac{\left(\mu_{k+1}-\mu_{k}\right)}{2} \frac{2 \pi}{N}}\right. \\
& \left.\left[\sum_{n=0}^{N-1} \sqrt{\varepsilon_{n} c_{n+1}} \cos \left((n+1 / 2)\left(\tilde{\mu}_{k+1}-\mu_{k}\right)\right)\right] / W\left(\cos \left(\tilde{\mu}_{k+1}-\tilde{\mu}_{k}\right), \beta\right)\right\} \cdot \prod_{\left\{x x^{\prime}\right\}} W\left(\cos \left(\tilde{\mu}-\tilde{\mu}^{\prime}\right), \beta\right)
\end{aligned}
$$

so that the variational energy $E_{\phi}(\beta)$ is given as averages of local operators with respect to a partition function of a spin model of the same global symmetry $z_{\mathrm{N}}$ in one less dimension $d=D-1$. The evaluation of (8) and (9) is a technical problem and can be performed in several ways, for instance by high and low temperature expansions with respect to the variational parameter $\beta^{3}$ ). The most convenient method however is that proposed in Refs. 4) and 7), consisting of embedding the operators to be computed in a small volume, subjected to the mean field boundary conditions which are appropriate to the effective model (7), in which all summations in Eqs. (8) and (9) are done explicitly. This method is in a certain sense equivalent to an expansion both in high and low temperature, which also seems to interpolate very well for intermediate couplings. The selfconsistency relation which has been used for the mean field for the effective model is a next to the nearest-neighbour of the type: 


$$
\begin{aligned}
& m=\left\langle\cos \mu \frac{2 \pi}{N}\right\rangle= \\
& =\frac{\left.\sum_{\mu=0}^{N-1} \cos \mu \frac{2 \pi}{N} \sum_{j=1}^{2 d} \sum_{\mu=1}^{N-1} \prod_{k=1}^{2 d} W\left(\cos \tilde{\mu}-\tilde{\mu}_{k}\right), \beta\right)\left[W\left(m \cos \tilde{\mu}_{k}, \beta\right)\right]^{2 d-1}}{\sum_{\mu=0}^{N-1} \frac{\sum_{j=1}^{d}}{\sum_{\mu-1}^{N-1}} \prod_{k=1}^{2 d} W\left(\cos \left(\tilde{\mu}-\tilde{\mu}_{k}\right), \beta\right)\left[W\left(m \cos \tilde{\mu}_{k}, \beta\right)\right]^{2 d-1}}
\end{aligned}
$$

where $j$ runs over the nearest neighbours and the power $2 d-1$ takes care of the next to the nearest neighbours treated with the mean field approximation. Expression (10) can be further simplified using the fact that, in this case,

$$
\sum_{\mu}\left(\sum_{j} \sum_{\mu_{j}} \prod_{k} \ldots\right) \equiv \sum_{\mu}\left(\sum_{\mu^{\prime}} \ldots\right)^{2 d}
$$

The volumes chosen for the calculations consist of the minimal one for the operator on the plaquette in Eq. (9), i.e., the plaquette, and in all the plaquettes belonging to the link for the operator of $\mathrm{Eq}$. (8), in order to have a consistently high temperature expansion. Summations in Eqs. (8) and (9) are done numerically, leading to the following expression for the variational energy:

$$
E_{\phi}(\beta, \lambda)=-\left\langle\frac{P+P^{+}}{2}\right\rangle-\lambda \frac{d-1}{2}\left\langle\frac{Q Q Q^{+} Q^{+}+h \cdot c \cdot}{2}\right\rangle
$$

We note that the coupling constant $\lambda$ is present only in an explicit way, which makes the analysis trivial with respect to $\lambda ;(d-1) / 2$ is the ratio between plaquettes and links. The dependence on the dimensionality is, on the contrary, non-trivial and the situation differs substantially from a non-gauge invariant variational or Lagrangian mean field treatment for which the dimensionality appears in front of the coupling constant and can therefore be eliminated by a rescaling, thus leading to features which are independent of the dimensionality.

The minimization of $E_{\phi}$ provides the following results. In four-dimensions we find for $Z_{2}, Z_{3}$ and $Z_{4}$ a first order transition between confining and free phases $^{8)}$, as appears in Fig. la, where $-E_{\phi}$ is reported as function of the variational parameter $\beta$ for the critical value $0.9<\lambda_{c}<1$ of the coupling constant (the exact value is $\lambda_{c}=1$ by self-duality). The first order nature of the transition is signaled by the sudden jump of $|\phi\rangle$ from the left maximum of $-E_{\phi}$ occurring at $\beta=\beta^{*}$ ) corresponding to a disordered state to the right partially ordered one.

\footnotetext{
*) $B$ is the critical value of the variational parameter for which the effective model undergoes a phase transition.
} 
It can further be proven that if the variational energy selects a state for which the effective model has vanishing magnetization (disordered state), correspondingly the wilson loop operator behaves with an area law corresponding to a confining situation, whereas if $m \neq 0$ (ordered state) there is a perimeter law. The discontinuity of the first order transition is also made evident by Fig. 1b, which shows the value $B_{m}$ of $B$ corresponding to the minimal energy (maximum probability) as function of $\lambda$. Only one curve is plotted for $Z_{2}$ and $Z_{4}$, because apart from the fact that $\beta_{4}^{*}=2 B_{2}^{*}=0.3672^{*}$ ), the two curves coincide within the computer digits preserving the fact that the $Z_{2}$ Hamiltonian splits into the sum of two independent $z_{2}$ ones ${ }^{6 *}$ ). The same numerical identity holds for a generic dimension $D$ giving confidence in the method.

For $\mathrm{N} \geq 5$ two second order ${ }^{* * *}$ ) phase transitions between confining massiess and massless ordered regimes appear in agreement with Monte Carlo calculations ${ }^{8}$, Refs. 10) and 11). The amazing fact is that $D \simeq 4\left(3.9<D_{N}<4.1\right)$ is the critical. dimensionality ${ }^{* * *}$ ) above which the low $\lambda$ transition, which in the limit becomes the $U(1)$ one, becomes of first order without any divergence of the correlation length forbidding the continuous limit of the theory; this coincides with the usual criterion of renormalizability of electrodynamics in the continuum. As a remark $Z_{N}$ gauge theories seems to be simpler than the $Z_{N}$ spin theories, for which the corresponding three phase structure in two-dimensions cannot be obtained by means of an unique simple ansatz but requires the use of a state which takes into account the structure of the domains and vortices 5$), 6)$. The quantity $\lambda_{\mathrm{Z}_{\mathrm{N}}}^{\mathrm{C}} \cdot \mathrm{N}^{2} / 2 \pi^{2}$ which tends to the $\mathrm{U}(1)$ critical coupling constant is shown in Fig. 3: after $\mathrm{N}>5$ the limit value of $\lambda_{\mathrm{U}(1)}^{\mathrm{C}}=1$ is pratically reached, indicating a common critical behaviour of $Z_{N}>5$ and $U(1)$. From this argument the low $\lambda$ transition $\lambda_{1}$ seems to be described in an excellent way; on the contrary the high $\lambda$ transition $\lambda_{2}$ does not satisfy very well the self-duality condition telling that $\lambda_{1} \cdot \lambda_{2}=1$. This can be due to the particular form for the coefficients $c_{p}(\beta)$ leading to the Wilson action. Between three and four dimensions at $D \simeq 3.2$ the $Z_{2}$ and $Z_{4}$ transition changes character from first to second order. In $D=3$ only the $Z_{3}$ model, equivalent to the three states Potts model, has a first order transition ${ }^{12)}, 4$ ).

\footnotetext{
*) See footnote on p. 4 .

**) This property also holds for the Lagrangian version ${ }^{9}$.

***) Actually the transition is not of the first order. ****) The critical dimensionality makes $\left.\frac{\partial^{2} E \phi}{\partial \beta}\right|_{\beta_{+}^{*}}=0$, as can be seen in the
plateau of $-E_{\phi}$ for $B \geq \beta^{*}$.
} 
For large $N$ the situation becomes less accurate since the effective model goes into the XY model and a mean field analysis no longer holds for $D-1=2$. Nevertheless, the fact that the $X Y$ model exhibits the Kosterlitz-Thouless transition ${ }^{13)}$, which is a transition without magnetization, suggests that the variational method tells that the $U(1)$ theory is always in a confining phase ${ }^{14}$; it would be interesting to study if the Kosterlitz-Thouless transition for the effective model implies a non-deconfining phase transition for the gauge theory or just a crossover, or if the dimensionality is too low for the application of the method.

We see that the variational approach provides a wide range of successful qualitative and quantitative results. Many things remain to be done: technical ones as the best choice for the variational state especially for large couplings (low temperature) or the introduction of matter fields ${ }^{7}$ ), others like the understanding of the structure of the ground state, or the importance of the fluctuations, which also seems to be small from Monte Carlo calculations, or the extension to non-Abelian gauge theories.

\section{ACKNOWLEDGEMENTS}

It is a pleasure to thank L. Masperi and R. Iengo for very fruitful discussions. 


\section{REFERENCES}

1) R. Balian, J. Drouffe and C. Itzykson, Phys. Rev. Dll (1975) 2104;

J. Drouffe, Nucl. Phys. B170 (1980) 211;

J. Drouffe, Phys. Lett. 105B (1981) 41;

E. Brezin and J. Drouffe, Saclay preprint DPL-T/81-57 (1981).

2) J. Greensite and B. Lautrup, Phys. Lett. 104B (1981) 41;

P. Cvitanovic, J. Greensite and B. Lautrup, Phys. Lett. 105B (1981) 197.

3) D. Boyanosky, R. Deza and L. Masperi, Phys. Rev. D22 (1980) 3034;

J. Cardy and M. Hamber, Nucl. Phys. Bl70 (1980) 79 .

4) L. Masperi and C. Omero, Trieste preprint IC/81/55 (1981), to appear in Nucl. Phys. FS

5) G. Aldazabal, D. Boyanosky, V. Baltar, L. Masperi and C. Omero, Phys. Rev. 24D (1981) 2274 .

6) R. Iengo, L. Masperi and C. Omero, ICTP preprint IC/81/200 (1981).

7) L. Masperi and C. Omero, Trieste preprint IC/81/172 (1981), to appear in Phys. Lett. C.

8) M. Creutz, L. Jacobs and C. Rebbi, Phys. Rev. D20 (1979) 1915.

9) H. Grosse, C.B. Lang and H. Nicolai, Phys. Lett. 98B (1981) 69.

10) S. Elitzur, R.B. Pearson and J. Shigemitsu, Phys. Rev. D19 (1979) 3698;

D. Horn, M. Weinstein and S. Yankielowicz, Phys. Rev. D19 (1979) 3715.

11) B. Lautrup and M. Nauenberg, Phys. Lett. 95B (1980) 63.

12) S. Knack Jensen and O. Moritsen, Phys. Rev. Lett. 43 (1979) 1736;

H. Blöte and R. Svenden, Phys. Rev. Lett. 43 (1979) 799;

H. Herrman, Z. Phys. B35 (1979) 171;

J. Kogut and D. Sinclair, Urbana preprint ILL-TH-81-17 (1981).

13) J.M. Kosterlitz and D.J. Thouless, J. Phys. C6 (1973) 118.

14) A.M. Polyakov, Phys. Lett. 59B (1975) 82; Nucl. Phys. B120 (1977) 429 ;

T. Banks, R. Myerson and J. Kogut, Nucl. Phys. Bl29 (1977) 493;

G. Bhanot and M. Creutz, Phys. Rev. Dl9 (1980) 2892. 


\section{FIGURE CAPTIONS}

Fig. 1 : a) Minus the variational energy $E_{\phi}$ for $Z_{2,3,4}$ as a function of the variational parameter $\beta$ for the critical value of the coupling constant $\lambda_{c}$ and $D=4$. The ground state jumps discontinuously, for increasing $\lambda$, from the left maximum to the right one.

b) The variational parameter $\beta_{m}$, which minimizes the energy $E_{\phi}$ as a function of $\lambda$ for $N=2,3,4$ and $D=n$.

Fig. 2 : a) Minus the variational energy at the critical dimensionality $D=4$ and for the critical value of $\lambda_{2}$ for which the maximum of $E_{\phi}$ starts to move to the right for increasing $\lambda$.

b) The variational parameter, which minimizes $E_{\phi}$, as function of $\lambda$. Discontinuities in the derivatives $\partial \beta_{\mathrm{m}} / \partial \lambda$ single out the second order phase transitions at $\lambda_{1}$ and $\lambda_{2}$.

Fig. 3 : The rescaled coupling constant defining the coupling constant of $\mathrm{U}(1) \lambda_{\mathrm{U}(1)}$ for $\mathrm{N} \rightarrow \infty$ as function of $\mathrm{N}$. For $\mathrm{N} \geqslant 6$ the limit value of $\lambda_{U(1)}=1$ is reached. 

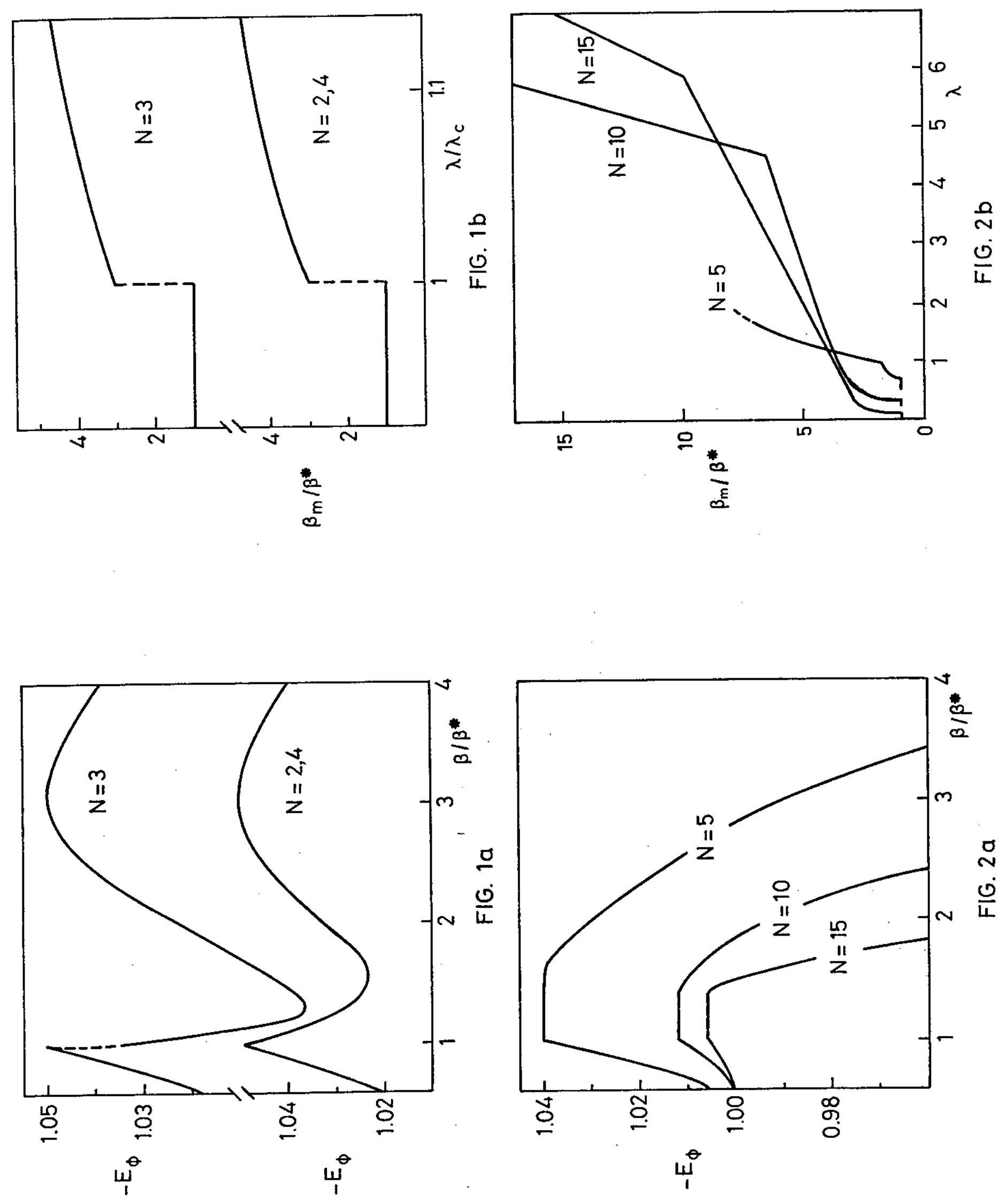


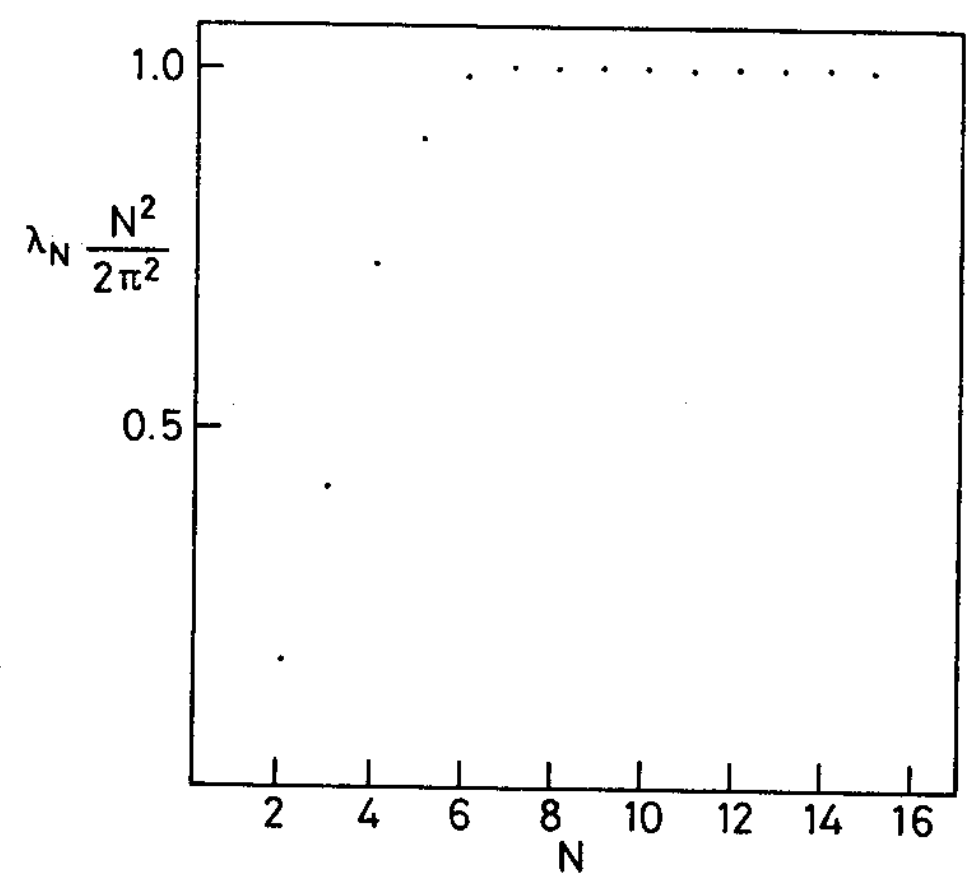

FIG. 3 